\section{Biosecurity in UK animal shelters}

\section{Siobhan Firmin, Emily Newbury, Marnie Brennan, Jenny Stavisky}

Centre for Evidence-based Veterinary Medicine, School of Veterinary Medicine and Science, University of Nottingham, Nottinghamshire, UK

\section{INTRODUCTION}

Infectious diseases are documented as being prevalent within animal shelters in the UK. This is due to a number of factors (host, pathogen and environmental) which predispose this animal population to infection. These diseases decrease the welfare and ultimately the adoptability of these animals, yet there is limited research into, and guidance describing, disease prevention and management. The aim of this study was to gain an understanding of the current disease prevention and management practices used by rehoming centres in the UK.

\section{METHODS}

An online questionnaire regarding infectious disease management was sent to 927 UK dog and cat rehoming organisations between October and November 2014. These were identified using a database generated by a previous study. One hundred and forty-six valid responses were received, giving a response rate of $15.7 \%$, and of these organisations $93(63.7 \%)$ had a rehoming centre. These rehoming centres reported that an estimated 20,589 dogs and 24,290 cats were rehomed by them in 2013.

\section{RESULTS}

Hand hygiene equipment was provided in most rehoming centres for animal care personnel and footbaths or footwear disinfection were the most common footwear hygiene precautions used (66.7\%). Only $19.4 \%$ of the rehoming centres reported that their animal care personnel had designated work clothes for use on-site only. Quarantine areas were available in $57.1 \%$ and $72.1 \%$ of the dog and cat rehoming centres respectively. Isolation areas were available in $65.1 \%$ and $80.3 \%$ of the dog and cat rehoming centres respectively. Approximately $92.0 \%$ of the rehoming centres vaccinated all or most of their animals, most commonly within 1-3 days of arrival. Puppies and kittens were housed separately from adult animals by $83.0 \%$ and $67.8 \%$ of the dog and cat rehoming centres respectively.

\section{SUMMARY}

This study demonstrated that animal rehoming centres in the UK are currently applying a wide range of biosecurity practices. It has also shown that the type and quantity of those practices used varied greatly between rehoming centres, especially when comparing large organisations with smaller individual animal shelters. These results indicate the need for accessible guidance describing how rehoming centres can best use their limited resources to reduce infectious disease prevalence. Therefore, more research is needed to evaluate the efficacy of each biosecurity practice in order to make accurate recommendations of the most suitable interventions for individual rehoming centres. 